\title{
Performance of WDM Transport Networks
}

\author{
Ezhan Karasan, Member, IEEE, and Ender Ayanoglu, Fellow, IEEE
}

\begin{abstract}
Wavelength division multiplexed point-to-point transport is becoming commonplace in wide area networks. With the expectation that the next step is end-to-end networking of wavelengths (in the optical domain without conversion to electronics), there is a need for new design techniques, a new understanding of the performance issues, and a new performance evaluation methodology in such networks. This paper describes approaches to that end, summarizes research results, and points to open problems.
\end{abstract}

Index Terms - All-optical networks, communication routing, network restoration, transparent optical networks, wavelength conversion, wavelength division multiplexing.

\section{INTRODUCTION}

W AVELENGTH division multiplexing (WDM), also known as optical frequency division multiplexing, is the use of multiple wavelengths to transmit different data streams. It is similar to frequency division multiplexing (FDM) except that in WDM, channel spacings are vastly larger than the information bandwidth of the signal. WDM is attractive since the same fiber can be employed for multiple data streams, saving costs on fiber and installation. Since an enormous bandwidth is available on fiber, WDM can provide an optical transmission system with an extremely large data rate: as of this writing, transmission systems with 50 wavelengths, each with a transmission rate of $20 \mathrm{~Gb} / \mathrm{s}$, have been demonstrated [1]. As such, the WDM technology is very attractive for networks that carry very high transmission rates, such as long-distance transmission networks. WDM has already been deployed in the backbone networks of all major long-distance carriers in the United States as a pointto-point transmission technology [2]. When WDM networks are deployed as such, every network node performs opticalto-electrical (O/E) and electrical-to-optical (E/O) conversions and switching is performed in the electrical domain. The explosive growth of the Internet and other communications services ensures that WDM deployment will accelerate in the near future.

As more traffic is carried on WDM networks, it will be desirable to reduce the number of $\mathrm{O} / \mathrm{E}$ and $\mathrm{E} / \mathrm{O}$ conversions in the network. The ultimate goal is to connect wavelengths on an end-to-end basis, where a wavelength goes through the

Manuscript received July 16, 1998; revised April 1998. This work was supported in part by DARPA under the MONET (Multiwavelength Optical Networking) Consortium of AT\&T, Lucent Technologies, Bellcore, Bell Atlantic, Pacific Telesis, and SBC, with the participation of NRL and NSA.

E. Karasan was with AT\&T Labs-Research, Red Bank, NJ 07701-7033 USA. He is now with the Department of Electrical and Electronics Engineering, Bilkent University, 06533 Ankara, Turkey.

E. Ayanoglu is with Bell Labs, Lucent Technologies, Holmdel, NJ 077333030 USA.

Publisher Item Identifier S 0733-8716(98)05734-5. network without $\mathrm{O} / \mathrm{E}$ and $\mathrm{E} / \mathrm{O}$ conversions. This process is known as wavelength routing, the connections are sometimes termed lightpaths, and the networks are known as transparent networks or all-optical networks. To this end, a series of largescale projects with partial U.S. Government funding [3]-[8] and partial E.U. funding [9]-[15] have been carried out. In such a network, when a connection is established, it goes through the network in optical form. One method to accomplish this is by using only wavelength add/drop multiplexing for the initial and terminal nodes on the connection, and without demultiplexing/multiplexing any of the wavelengths at intermediate network nodes. The elements in the network nodes of such architectures are known as the fiber crossconnects (FXC's). Clearly, this technology cannot employ fibers in an efficient manner since full-fiber connections would be needed between end-to-end source destination pairs. In order to improve wavelength packing onto fibers, consideration is being given to demultiplexing individual wavelengths on network fibers inside network nodes, and to remultiplexing wavelengths to fibers in order to improve fiber utilization. This can be accomplished by using wavelength selective crossconnects (WSXC's) which do not employ any wavelength conversion. An alternative is wavelength interchanging crossconnects (WIXC's) which employ wavelength conversion to pack more data-bearing wavelengths onto existing fibers in the case of wavelength conflicts.

\section{A. Wavelength Conversion}

Clearly, employment of wavelength conversion reduces the blocking probability for a given utilization and size of a crossconnect. For this reason, based on intuition, such crossconnects are considered desirable, and sometimes even unavoidable [16]-[18]. There exists some evidence in the literature that there may be significant performance gains by using wavelength conversion. On the other hand, there is other evidence in the literature that illustrates that the operating regions of very large gain due to wavelength conversion are not very large and can be made even smaller by using better wavelength selection and routing algorithms or more fiber in the network. This overview paper outlines these approaches in detail.

Although in this paper we conclude that the blocking performance versus utilization advantages of wavelength conversion are limited, there may be advantages to having wavelength conversion in the network for simplifying operation, administration, and management (OAM) [19]-[21]. Although there exist several candidate technologies to implement optical wavelength converters without any electrical conversions, all of these technologies are currently at the stage of development. 
Consequently, it is difficult to quantify the advantages of optical wavelength conversion due to simplicity in OAM, especially because of the unavailability of any actual information on wavelength converters, such as their cost and performance (power loss, bit error rate, etc.). WSXC's are becoming available together with their OAM software. When commercially deployable WIXC's become available, it will be possible to make price, reliability, and simplicity comparisons, and to determine if the lack of performance advantage can be overcome by these criteria.

Another advantage sometimes cited is the unfair behavior of simple-minded routing and wavelength selection algorithms, resulting in shorter paths receiving higher priority [22], [23]. However, this problem can easily be solved by employing routing and wavelength selection algorithms that take fairness into account rather than resorting to expensive wavelength conversions. This will be elaborated later in Section II-B3.

It is a generally held belief that if there will be some wavelength conversion in the network, the best location is the network boundary. It will be shown in Section II-D that some wavelength converters in the network can effectively capture most of the blocking advantage due to wavelength conversion. However, the presence of more than one entity to manage may not be very desirable from OAM and inventory management points of view and, again, even this need is questionable.

1) Relation to Time Slot Interchanging: In time division multiplexing (TDM) networks, the basic switching element is a TST switch, where the $T$ blocks are multiplexer and demultiplexers in time which may include time slot interchangers, and $S$ is a space switch. The time slot interchange concept was introduced [24], [25] to reduce the high internal blocking loss due to assigning the originating and terminating lines to the same time slot [26, ch. VIII-2]. By making an analogy to WDM networks, it is sometimes argued that wavelength converters are thus needed to implement nonblocking WDM crossconnects. The concept of nonblocking here refers to the certainty of assigning a time slot (wavelength) in the originating line to a time slot (wavelength) in its destination line if there exists an available time slot (wavelength) in that line. It is important to note that this capability does not ensure a nonblocking network or a certainty in assigning a time slot (wavelength) to its destination in the network; it merely ensures that the blocking probability in such a network will be less than that in a network without time slot interchangers (wavelength converters). In the absence of such interchangers or converters, the reduction of blocking probability in the network can be achieved by means of sophisticated routing and time slot or wavelength selection algorithms, although the blocking performance of the network with such converters would always be a lower bound than on that of a network without such converters. In TDM networks, employing such an algorithm on a call-by-call basis can be prohibitive. In addition, time slot interchanging is achieved by simple memory elements. In WDM networks, however, such algorithms need to be employed only in the provisioning stage for aggregated connections and considering the potential

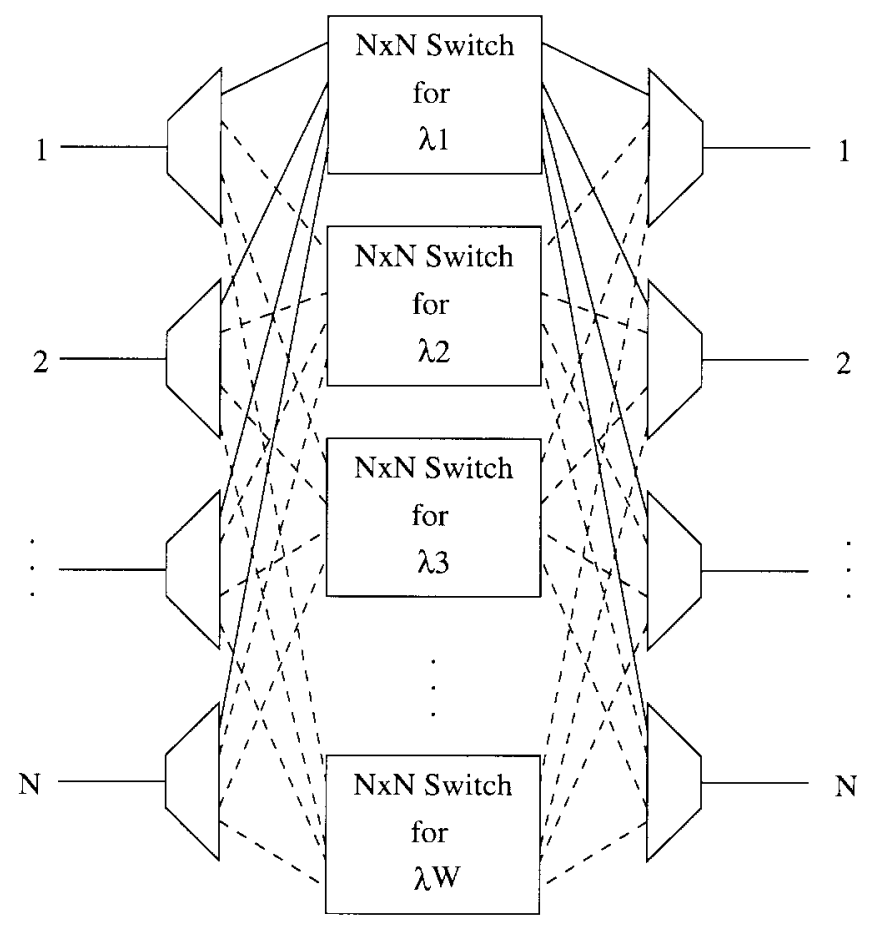

Fig. 1. Wavelength selective crossconnect (WSXC).

high cost of wavelength conversion, the prospect of using such algorithms, even with the associated need to somewhat overengineer the network can be preferable. We will quantify the performance tradeoffs associated with this set of design choices in Section II.

2) Wavelength Conversion Technologies: Similar to the case in TDM networks where switching is accomplished by means of TST structures, WDM switching can be accomplished by means of WSW structures, where the first $W$ stage demultiplexes each fiber into constituent wavelengths, the S stage performs space switching for each wavelength, and the second $W$ stage multiplexes wavelengths onto different fibers. Demultiplexing and multiplexing wavelengths is a relatively simple task; various structures for this purpose exist and are used in wavelength add-drop multiplexers (WADM's). For space switching of wavelengths, again, various structures known as wavelength routers exist. One such structure is obtained by using a layered switch with $\mathrm{LiNbO}_{3}$ technology [27]. The resulting architecture is known as a WSXC. A block diagram of a WSXC is shown in Fig. 1.

By replacing the wavelength routers in WSXC with one that can change wavelengths during the routing process, a WIXC is obtained (Fig. 2). Depending on where the wavelength converters are located and their capabilities, this structure can implement fixed-input-to-variable-output, variable-inputto-fixed-output, or variable-input-to-variable-output switching. The most desirable structure is the variable-input-to-variableoutput switch.

Wavelength conversion techniques can be classified into the following categories [28], [29].

1) Optoelectronic wavelength conversion: This technique is based on the electrical detection of the incoming signal and its subsequent optical modulation onto a wavelength. 


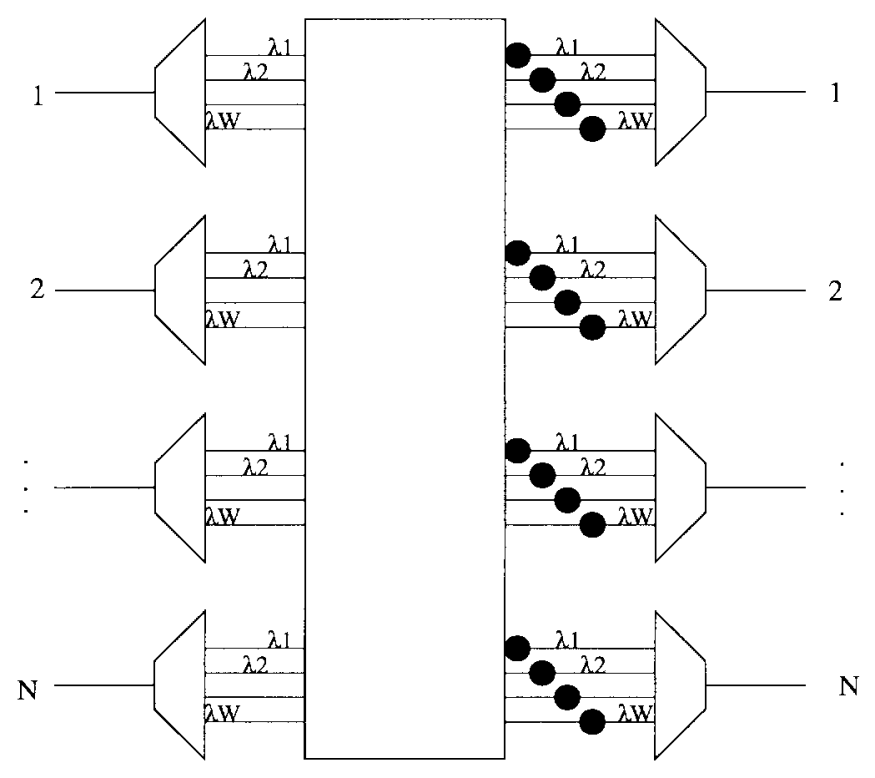

Fig. 2. Wavelength interchanging crossconnect (WIXC).

Unless tunable lasers or laser arrays are used, this is a variable-input-to-fixed-output technique. It is mature, but is not preferable due to its nontransparent nature.

2) Optical gating wavelength conversion: This technique is based on a device that changes its characteristics based on the intensity of an optical carrier signal, known as a probe. The output has information modulated onto the probe. There are many, perhaps countless, methods to achieve the desired effect. Some examples are crossgain modulation in semiconductor optical amplifiers, crossphase modulation in semiconductor optical amplifiers, and nonlinear optical loop mirrors. An advantage is the filtering of noise. This technique, however, is also limited to variable-input-fixed-output systems.

3) Wave-mixing wavelength conversion: This technique is the least explored. It includes optical-acoustic wave mixing, optical-electrical wave mixing, and nonlinear optical wave mixing. The first two are considered unlikely to provide the large frequency translation required for WDM. The nonlinear optical wave-mixing results from nonlinear interactions among the optical waves present in a nonlinear optical material. This mechanism is sensitive to both amplitude and phase, and can thus achieve transparency, i.e., the process is variableinput-variable-output. Although various techniques such as four-wave mixing in passive waveguides, four-wave mixing in semiconductor optical amplifiers, and difference frequency generation are being explored, this technology is not mature.

Space limitations prevent us from a detailed discussion of various wavelength conversion technologies. For the purposes of this paper, we would like to emphasize that although wavelength conversion in a transparent manner seems plausible, at this time technologies to achieve that goal in commercial products are not yet available. Also, there is a widely held belief that when they become available their cost will be a significant consideration.
B. The Role of WDM in Wide Area Networks (WAN's), Metropolitan Area Networks (MAN's), Local Area

Networks (LAN's), and the Access Loop

WAN's, especially the long-distance network, are where the application of wavelength routing is most appropriate: the transmission rates are very high, are continuously increasing due to the explosion of new applications such as the Internet, and the placement of new fiber is very expensive. Most of the current emphasis on WDM is in wide area networking, both from equipment manufacturer as well as service provider viewpoints. Also, wide area networking, due to the aggregation of millions of users, is where the economies of scale in introducing new technologies is the highest. Currently, all of the major long-distance network providers have point-to-point WDM in their long-distance networks. Currently, however, there is no wavelength-routing technology implemented in any long-distance network provider's network. Because of the reasons outlined above, it can be expected that wavelengthrouting technologies in WDM will first take place in longdistance networking. Most WAN's have mesh networks which are well-suited to wavelength routing.

MAN's can be considered as small-size WAN's. Significant differences are: 1) MAN's carry much less traffic as compared to WAN's; 2) typically have either nonmesh or limited meshlike topologies without connectivities as rich as WAN's; and 3) placement of fiber may sometimes be relatively easier, following existing conduits for public works infrastructure. These make economic-based arguments for WDM networking less valid in the case of MAN's. With the explosive growth of the Internet, however, and with the relative ease of networking different sources with different formats (transparency) there can be advantages to WDM networking in MAN's, or networks whose sizes are about as large as metropolitan areas; perhaps somewhat larger (an aggregate network for several small suburban towns) or somewhat smaller (about the size of a university or a large company campus). Currently, there is interest in U.S. Government funding agencies in pursuing WDM networking research into such networks.

Although following the logical process in the two paragraphs above seems to lead to the conclusion that wavelength routing is not cost-effective for LAN's, there exists a commercially available WDM LAN [30]. This product is based on a research project named Rainbow [31]. The raison d'etre for this product is cited as protocol transparency: it can interconnect computers and computer peripherals which employ different protocols such as FDDI, T3, token-ring, HIPPI, etc. Although the future product plans of the manufacturer on similar products are unclear, this product shows that there can perhaps be some room for wavelength routing in LAN's.

Currently, the access networks in the world are about to undergo an extensive overhaul. Driven by the demand for Internet access and the improvements in compression, transmission, and switching technologies, the access loop will undergo a transmission speed overhaul of about two-three orders in magnitude. Although for individual users, copper-based technologies seem sufficient to carry these new transmission speeds throughout the next decade, for aggregated traffic, fiber- 
based transmission may have a place [fiber to the curb (FTTC) and the associated very high-speed digital subscriber line (VDSL) technologies are based on this premise]. When these speeds need to increase, there may be some room for WDM, and eventually WDM networking in the access network, but the authors believe that the access loop can only be the last fortress to be captured by WDM networking.

Computer backplanes are small-size and large-capacity networks. The implementation of WDM links and WDM networking for computer backplanes will be attractive if the interprocessor transmission speeds reach the rates of multi$\mathrm{Gb} / \mathrm{s}$. However, the WDM technology is essentially a circuitswitching technology. It is not well-suited to packet transmissions needed for computers and their backplanes.

\section{Network Topologies}

Various topologies for all-optical network have been considered in the literature. The two most important are ring networks and mesh networks. A typical WAN is in the form of a mesh as far as the basic structure is concerned. Rings are suitable for LAN's and MAN's. Some combinations of meshes and rings have also been seriously considered and deployed; for example, the ring-of-rings architecture [32] and the mesh-ofrings architecture [33]-[35]. A network that is studied in the literature but does not really have a practical implementation consists of families of several multidimensional networks, such as the mesh-torus [36], [37]. Due to the multidimensional structure, these networks have a very high degree of connectivity, resulting in a relatively small number of shared links among paths for the same source-destination pair. As will be described later in the text, this phenomenon results in a very high utilization gain for wavelength conversion at small loads. At least for WAN's and MAN's, however, implementation of multidimensional networks is not practical, and therefore can be ruled out. As will be stated later in the text, mesh networks tend to have higher wavelength conversion gain as compared to ring networks. In addition, mesh networks have higher conversion gain than fully connected networks. We will quantify the wavelength conversion gains for mesh networks later in the text, but we would like to point out here that there has been significant debate on the value of this quantifiable gain in the optical networking community.

\section{Data Networks Versus the Transport Network}

Computer communications networks or data networks, typically employed for LAN applications, and backbone telecommunications networks or transport networks, typically employed for the MAN and the WAN, are different. The difference is mainly due to the aggregation needed in the transport network.

In the data network, a call or a connection request, or for datagram networks, a packet transmission, is responded to by evaluating whether there are sufficient resources available and, if yes, by accommodating the request. The network is designed to attain a certain blocking probability at a certain utilization level. The operating point in the network for typical utilization levels is chosen with respect to the statistics of expected traffic types where the peak-to-average ratios of traffic levels can be very large. As such, the design of a data network involves statistical modeling of the source, and the individual calls are individually routed in an incremental manner while other traffic exists in the network. In the meantime, some calls may terminate, thus releasing some of the existing network resources. The network design is accomplished by trying to minimize a blocking probability consideration for a statistical model of the sources while they dynamically use the network.

In the transport network, individual calls do not result in rerouting, but are placed on provisioned routes determined during network planning. The planning of the network is made based on an aggregate demand pattern and its forecast future values. Although there are hourly, daily, or weekly changes to this pattern due to aggregate demand changes, there is, at most, a small variation in the network due to individual calls. The provisioned network design is accomplished by considering the existing resources as a transport network and solving a multicommodity flow-maximization problem. There is no statistical modeling of the sources and there is not a blocking-probability consideration. This formulation leads to an integer programming problem. Typically, the demand from the network does not change due to statistical differences around a mean, but shows an increase due to an increase in population and demand for new services.

The two formulations, however, are related. Although the transport network design problem should ideally be formulated using the second method outlined above, general considerations on the advantages of wavelength conversion can be evaluated using both techniques. Although this is not a formal claim, it can be expected, based on general considerations, that general observations made on the advantage of wavelength conversion using one design methodology will not be significantly different as long as both design methods are employed appropriately in their own right. In this paper, we will use the results employing both formulations and will qualify the method employed each time a result is presented.

\section{DESIGN AND PERFORMANCE ANALYSIS OF WDM NETWORKS}

In a WDM network there are mainly two problems to solve. These problems are coupled, but are generally treated independently. The first problem is that of network design. The inputs to this problem are a network traffic demand (or perhaps a family of such demands) and, possibly, a general network topology (most long-distance network providers have existing fiber plants that are not likely to change with respect to demand variations; as more traffic comes into the network, new optical amplifiers are placed on more dark fibers that become lit). The output or the solution of this problem determines the network configuration in terms of the number of fibers, the size of crossconnects, needed optical amplifiers, adddrop multiplexers, etc. Some of the existing work in the literature pursues minimization of the number of wavelengths for this problem. On the other hand, in most practical cases, the number of wavelengths per fiber is determined by the technology and is fixed. If a single fiber does not suffice to 


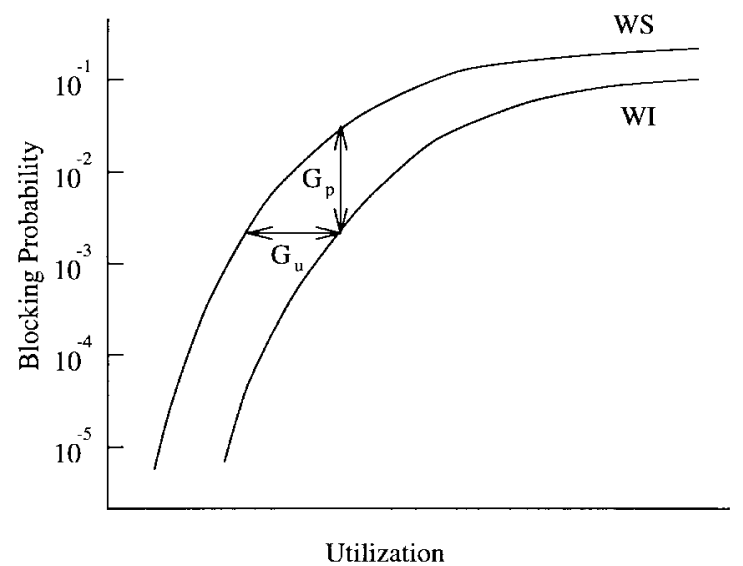

Fig. 3. The definitions of utilization gain $G_{u}$ and blocking gain $G_{p}$.

carry existing traffic on a link, more fibers on that link need to be lit. As such, minimization of the number of fibers, fiber miles, the size of crossconnects, etc., or a combination of the above, are more meaningful measures of the merit of a design. The ultimate measure of merit, of course, the cost of a network, which can be obtained as a combination of the number of fibers, fiber miles, etc., although its optimization is not necessarily a straightforward task. We will elaborate on possible approaches and resulting techniques later in the sequel.

As stated above, the second problem in WDM networks is that of network operation. In this case, for a given network (topology and resources) and for given demand, routing and wavelength selection are carried out. Typically, average values of traffic demand are known or forecast while the actual demand changes randomly. Therefore, the possibility of blocking in the network exists. A measure of the quality of a routing and wavelength selection algorithm is the blocking probability for a given utilization in the network. As stated before, the performance of networks with wavelength conversion, called wavelength interchanging (WI) networks, provides a lower bound than those without, called wavelength selective (WS) networks, as shown in Fig. 3. Therefore, there is always a wavelength conversion gain. This gain can be quantified as the utilization gain:

$$
G_{u}=\frac{\text { Offered Load with WI }}{\text { Offered Load with WS }}
$$

or the blocking gain

$$
G_{p}=\frac{\text { Blocking Probability with WS }}{\text { Blocking Probability with WI }} .
$$

Both quantities are larger than or equal to unity. The blocking gain $G_{p}$, since it is the ratio of probabilities, is expressed best as orders of magnitude. Values of $G_{p}$ that are less than about an order of magnitude are not considered significant. Similarly, values of $G_{u}$ that are less than about 1.1 are not considered significant.

These performance metrics have been evaluated in the literature with different types of traffic demand. The traffic demand is classified according to time dependence: static or dynamic. The dynamic traffic demand case is further divided into two classes: 1) connection requests arrive one at a time, which has attracted almost all of the attention so far and 2) batch connection arrival processes which have been considered recently. Batch arrivals are a good model for demands to establish virtual networks. They also represent the group of individual or batch arrivals occurring between two successive reconfiguration times of a network. The algorithms and analysis techniques for the static and dynamic traffic demands are presented in Sections II-A and II-B, respectively. The restoration problem for WDM networks is studied in Section II-C. The performance of WDM networks are evaluated for networks that have wavelength conversion only at selected nodes (sparse conversion) and for networks for which wavelength converters have a limited conversion bandwidth (limited conversion). The effects of sparse and limited wavelength on network performance are discussed in Sections II-D and II-E.

\section{A. Static Traffic Demand}

The first problem studied in the literature is the static traffic demand. Demands are predetermined and the network is designed to carry this traffic. When the network exclusively employs wavelength converting switches, this problem is identical to the network design problem in circuit-switched networks [38]. When the network has no wavelength converters, this problem is called the static lightwave establishment (SLE) problem by Chlamtac et al. [39]. This problem can be formulated as an integer programming (IP) problem with different objective functions. One possible choice is to minimize the number of wavelengths necessary to carry all demands, and it is shown by Chlamtac et al., that the SLE problem is NP-complete.

Several heuristics have been proposed for the SLE problem, and their performances have been compared with the wavelength interchanging case which gives a lower bound on the number of wavelengths. Using an intuition observed in task scheduling problems the paths are sorted according to their hop counts, and are assigned wavelengths starting from the longer paths [39]. This algorithm is known as the Longer Paths First policy. The rationale for this heuristic is the difficulty of finding an idle wavelength on a large number of links when establishing long connections in a heavily loaded network. The algorithm assigns wavelengths to connections sequentially, and the number of wavelengths is incremented when a connection cannot be established on any of the currently existing wavelengths. It is demonstrated that the difference between the number of wavelengths with and without wavelength conversion is less than $2 \%$ for a mesh network with ten nodes [39].

Other heuristic wavelength assignment algorithms have been proposed for static demand network design. Sato et al. [40]-[42] used the longer-paths-first policy to accommodate paths. The difference between the number of wavelengths with and without wavelength conversion is found to be less than $4 \%$ for various traffic volumes at a 50 -node mesh network.

The effect of physical connectivity of the network with the minimum number of wavelengths necessary to carry a 
TABLE I

Complexities of the Multicommodity Flow Problem Formulations

\begin{tabular}{c||c|c}
\hline \hline & Flow Formulation & Path Formulation \\
\hline \hline number of variables & $N(N-1) M W$ & $N(N-1) k W$ \\
\hline number of constraints & $M W+N^{2}(N-1) W+N(N-1)$ & $M W+N(N-1)$ \\
\hline \hline
\end{tabular}

given traffic demand is studied by Baroni and Bayvel [43]. The parameter $\alpha$ denotes the number of links in the network and the number of links in the fully connected topology. The number of wavelengths without wavelength converters is computed using a heuristic algorithm based on a shortest path routing and longer paths first wavelength assignment algorithm. This is compared to the number of wavelengths with wavelength conversion which is given by the limiting cut in the network. The results obtained by using several existing topologies as well as randomly generated mesh networks show that wavelength conversion does not provide any significant reduction in the number of wavelengths. Furthermore, the number of wavelengths strongly depend on $\alpha$, and the wavelength conversion gain is maximum for the intermediate values of $\alpha$ as shown by Jeong and Ayanoglu [44], e.g., for fairly connected mesh networks. The wavelength conversion gain for networks with small (ring) and large (fully connected) values of $\alpha$ is very limited.

Minimizing the number of wavelengths can result in systems with unrealizably large number of wavelengths, especially when the traffic volume is large. This can be a problem since the number of available wavelengths with current WDM technologies is relatively small. This led researchers to reformulate the static network design problem with a bounded number of wavelengths. Different objective functions have been considered. Ramaswami and Sivarajan formulated the problem as the maximization of the carried traffic [45]. This problem is equivalent to the multicommodity flow problem that maximizes the throughput through a network [46].

Given the traffic demand and the number of fibers per link, Wauters and Demeester [47] presented different formulations for the multicommodity flow problem with and without wavelength conversion. The first formulation is called the flow formulation which considers all possible paths between a source-destination pair. The second formulation considers a small and fixed number $k$ of shortest paths between any source-destination node pair, and it is called the path formulation. Although the limited number of paths in the path formulation may potentially reduce the carried demand, the numerical studies show very little penalty [47]. This is largely due to the fact that shorter paths are more likely to be a part of the optimum solution than the longer paths. The computational requirements of these formulations were also compared. The number of variables and constraints in these formulations are shown in Table I with a full mesh demand, where $N$ is the number of nodes, $W$ is the number of wavelengths, and $M$ is the number of links.

The number of variables and constraints with the flow formulation are at least $N$ times larger than the ones with the path formulation. With the large complexity of solving IP problems, the flow formulation is not scalable to networks with large number of nodes and wavelengths.

Nagatsu et al. [48] presented a more realistic formulation of the static network design problem that minimizes the number of fibers in the network such that the network is able to carry a given traffic demand. A heuristic algorithm is proposed for minimizing the total number of ports (both inter and intraoffice) in the network. The ratio of ports with and without wavelength conversion has been computed with different number of wavelengths and different traffic demand in a mesh network with 15 nodes. This ratio is less than $15 \%$ at $W=8$ and less than $35 \%$ at $W=32$. It is also noted that these ratios decrease with increasing traffic, i.e., the benefits of wavelength conversion decreases with the total number of ports in the network. In this case, the wavelength conversion gain is higher than in the case with unlimited number of wavelengths. The gain is relatively small, however, for a network carrying a large amount of traffic with a moderate number of wavelengths.

\section{B. Dynamic Traffic Demand}

The problem of dynamically establishing and terminating connections using fixed network resources has been studied extensively in the literature. The case of dynamic traffic demand models several situations. First, it may become necessary to reconfigure the network in response to changing traffic patterns or link/node failures. Second, with the rise in broadband traffic it is expected that the leased-line rates for private virtual networks and Internet service provider links will reach $2.5 \mathrm{~Gb} / \mathrm{s}$ and higher. The demand for such services will change with time, not only because the traffic demands of the customers are changing with time, but also because the demand for such services is predicted to grow rapidly. We have to emphasize, however, that the dynamic nature of these traffic demands will occur on a much larger time scale compared to traffic in today's public switched telephone networks: the connections may last for hours, days, or even weeks. Since the statistics of the traffic for a nonexistent service are not available, all of the studies presented here make the assumptions of Poisson arrival processes and exponentially distributed holding times.

The most often used performance measure for the dynamic connection establishment problem is the blocking probability. The general belief that the benefits of wavelength conversion increase considerably when the demands are dynamic is partially verified by the results in the literature. The conversion gains reported in these studies are larger than the corresponding numbers with the static demand. The increase is not substantial, however, and the conversion gain decreases rapidly with the traffic volume for which the network is designed. In the rest of the section we present some of the results in the literature, and discuss how topology, network size, traffic volume, routing, and wavelength assignment algorithms affect the wavelength conversion gain.

1) Routing and Wavelength Selection Algorithms: The problem of dynamic connection establishment in optical networks without wavelength converters is first discussed 
by Chlamtac et al. [39]. This algorithm decouples the routing and wavelength assignment problems: In fixed routing, a fixed path is used each time a connection is to be set up. Once the routing problem is solved, the wavelength is selected, based on several heuristics, from among the available wavelengths. In order to reduce the number of wavelength conflicts, a greedy algorithm called the first-fit algorithm is proposed [39]. The set of wavelengths are assumed to be indexed arbitrarily, and each connection is established on the available wavelength with the smallest index. The rationale behind this algorithm is to pack connections over smaller indexed wavelengths so that finding an available wavelength later is easier.

The performance of the first-fit algorithm is compared with the random wavelength selection algorithm in which a wavelength is selected at random among available wavelengths. It is shown through simulations that the blocking probability of the first-fit algorithm with fixed routing is considerably lower than the blocking probability with the random wavelength selection algorithm [37], [49], [50]. This is especially true at low network utilizations and when the main cause of blocking is wavelength conflicts. The ratio of blocking probabilities with the first-fit and random algorithms for mesh networks is $3-10$ [37], [50], and the ratio of network loads to achieve the same blocking probability is $1.6-1.8$ [49].

The blocking performances of wavelength interchanging and wavelength selective networks are compared by Kovacevic and Acampora [37] for the mesh-torus network with fixed shortest path routing and first-fit wavelength assignment algorithm. For a $101 \times 101$ mesh-torus network with $W=5$, wavelength conversion, gains of $G_{p}=10^{4}$ at an average network utilization of $12 \%$, and $G_{u}>5$ at $P_{B}=10^{-3}$ are obtained. A conversion gain of $G_{p}=10^{8}$ at an average utilization of 3\% was obtained by Subramaniam et al. [51], with a $101 \times 101$-mesh-torus network and $W=8$. The specific routing algorithm for the mesh-torus produces a large number of intersecting paths, and the paths in the mesh-torus topology are fairly long. Because of these reasons very high conversion gains are obtained with the mesh-torus topology. The effects of the topology and the routing algorithm are discussed in more detail in the next section.

The idea of packing wavelengths is further extended in the most-used algorithm in which the available wavelength that is used most in the network is selected [52]. Instead of the predetermined order that the first-fit algorithm uses, the most-used heuristic packs the wavelengths according to their utilizations. It is shown that the most-used algorithm performs slightly better than the first-fit algorithm for the ring topology [53]. Their performances, however, are very close for the mesh-torus topology [53]. This is largely due to the fact that while a large number of paths intersect in the ring, the probability that two paths intersect in a mesh is small. Selecting the most used wavelength does not reduce the blocking much in a mesh since the high usage on a wavelength may result from the usage in a completely independent part of the network.

A new wavelength assignment algorithm for fixed routing, called max-sum $(M \Sigma)$, was recently proposed by Subramaniam and Barry [53]. Given a route, the $M \Sigma$ algorithm chooses the wavelength that maximizes the total network capacity after the connection is established. The network capacity $V(\beta)$ is defined as a sum over all path capacities, i.e.,

$$
V(\beta)=\sum_{p \in P} C(\beta, p)
$$

where $P$ is the set of all possible paths, and $C(\beta, p)$ is the capacity of path $p$ defined by

$$
C(\beta, p)=\sum_{\lambda} \min _{l \in p} C_{l}-U_{l, \lambda},
$$

where $C_{l}$ is the number of fibers on link $l$, and $U_{l, \lambda}$ is the number of fibers on link $l$ for which wavelength $\lambda$ is utilized in state $\beta$. The $M \Sigma$ algorithm computes the total network capacity for each new network state corresponding to all possible wavelength selections, and chooses the one that results in the maximum capacity.

The $M \Sigma$ algorithm performs considerably better than other wavelength selection algorithms (random, first-fit, and mostused) with fixed routing in ring and mesh-torus topologies [53]. The worst case time complexity of the $\mathrm{M} \Sigma$ algorithm is $O(|P| M W)$, where $|P|$ is the number all possible paths, whereas the worst case complexities of the random, first-fit, and most-used algorithms are all $O(M W)$.

Ramaswami and Sivarajan [45] used alternate routing to evaluate the benefits of wavelength conversion. The set of shortest paths and the set of wavelengths are preordered. A connection request is routed over the first available path, and the first-fit algorithm is used for wavelength selection. The utilization gain is computed at $P_{B}=10^{-2}$ for randomly generated mesh networks: $G_{u} \approx 1.0$ for a 16 -node network and $G_{u}=1.4$ for a 1000 -node network $(W=10-32)$.

The blocking probability is reduced substantially when an adaptive routing algorithm is used which chooses the least congested path among a set of alternate paths; e.g., the set of $k$ shortest paths. The route and wavelength are selected in such a way that the blocking probability for future connection requests is reduced by distributing the traffic over less loaded parts of the network [50]. The routing and wavelength assignment problems can be solved either separately or jointly.

The least loaded routing (LLR) algorithm is proposed by Karasan and Ayanoglu [50] for optical mesh networks both with and without wavelength converters. In the case of a wavelength interchanging network, the LLR algorithm is similar to the real time network routing (RTNR) algorithm used in telephone networks [54]. With the LLR algorithm in a WS network, the route-wavelength pair that maximizes the residual capacity over all wavelengths and over $k$ shortest paths is selected jointly, i.e., LLR chooses the route-wavelength pair $(p, \lambda)$ that achieves

$$
\max _{(p, \lambda)} \min _{l \in p} C_{l}-U_{l, \lambda}
$$

where $C_{l}$ and $U_{l, \lambda}$ were defined above. Since the LLR algorithm only checks the path capacities for each possible route-wavelength selection, the computations can be done on the current state of the network. 


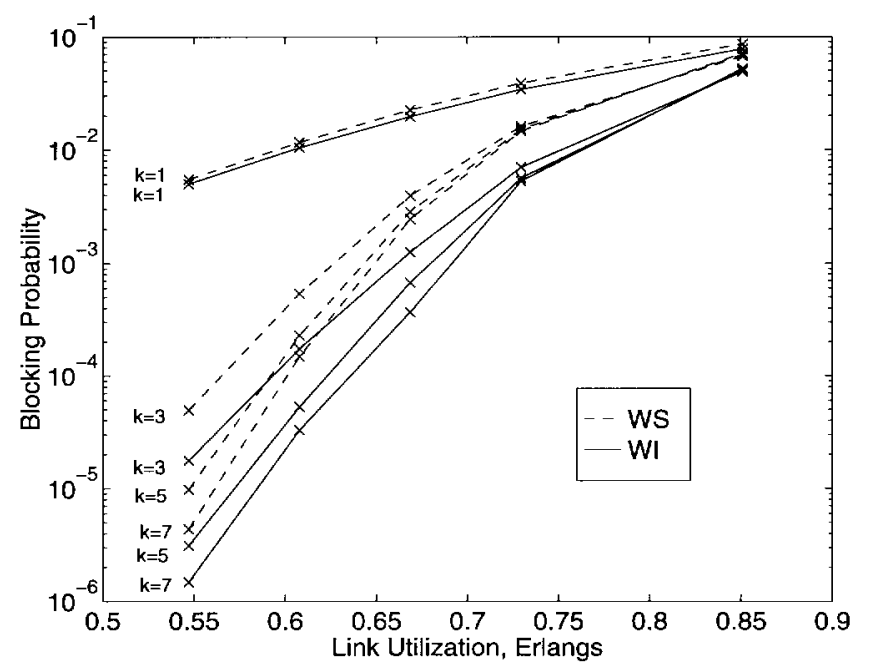

Fig. 4. Blocking probability versus network utilization with the LLR algorithm for $F=5.25$ and $k=1,3,5,7$.

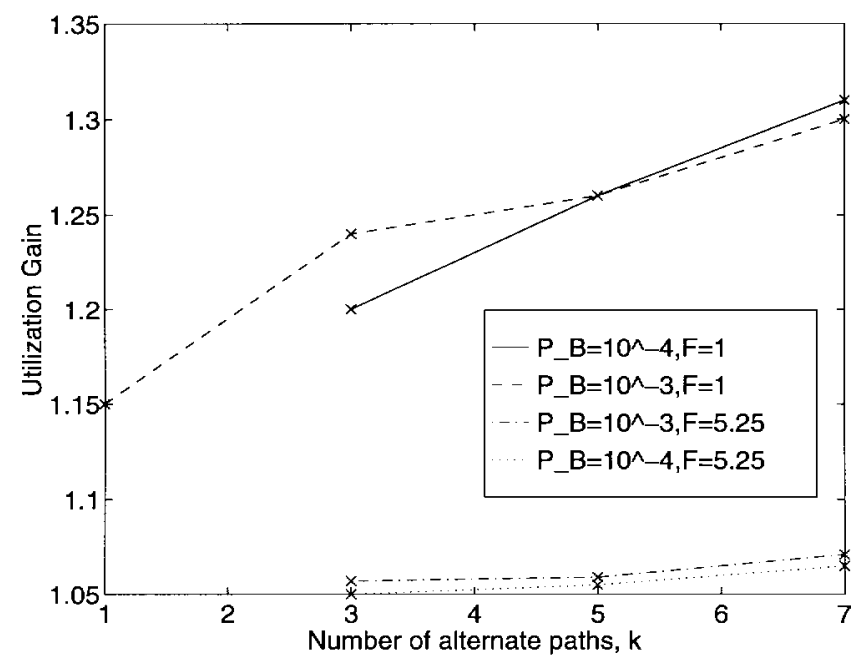

Fig. 5. $G_{u}$ versus the number of alternate paths with the LLR algorithm.

It is shown in Fig. 4 that the LLR algorithm reduces blocking for both WS and WI networks compared to shortest path routing $(k=1)$. These simulations were done using a 30 node mesh network which is an approximation of a national scale transport network in the United States. The number of wavelengths is eight. Furthermore, as shown in Fig. 5, with the LLR algorithm the wavelength conversion gain $G_{u}$ increases as $k$ increases [50]. This is intuitively expected since alternate paths may be longer than the shortest path, and longer paths create more wavelength conflicts. There are other reasons why the wavelength conversion gain increases with the LLR algorithm, and a theoretical framework justifying this fact will be discussed in the next section.

As pointed out in [53], the $M \Sigma$ algorithm can also be extended to adaptive routing using $k$ shortest paths. The network capacity is computed for each selection of the routewavelength pair. The worst case complexity of the adaptive $M \Sigma$ algorithm is $O(k|P| M W)$, whereas the worst case complexity of the LLR algorithm is $O(k M W)$ which is smaller by a factor proportional to $k N^{2}$. The complexity of the
$M \Sigma$ algorithm increases quadratically with $k$ (since $|P|$ is $O\left(k N^{2}\right)$ ), instead of the linear growth in LLR.

The blocking performance of WDM transport networks have also been studied by Karasan and Ayanoglu [55] when the traffic demand arrives in batches. In this study, the existing connections in the network are allowed to be rerouted in order to accommodate a larger number of connections arriving within the new batch. The number of blocked connections in a 30-node mesh network is reduced by an order of magnitude if the network is reconfigured each time a batch arrives. It is shown that the difference in blocking probability with and without wavelength converters is very small with the optimum reconfiguration of the network, and with tunable receivers and transmitters at the edges of the network. The penalty for not having wavelength converters within the network is the additional number of connections that need to be reconfigured (an increase of 3\% of all existing connections to $5 \%$ of connections). When there are no tunable receivers and transmitters (without wavelength converters the existing connections are kept at the same wavelength after rerouting), the benefit of wavelength converters increases: an increase from $G_{p}=1$ with full reconfigurability to $G_{p} \approx 2$ without tunable receivers and transmitters (limited reconfigurability).

2) Analysis Techniques for Blocking Probability: Analysis of the blocking probability in WS networks has been studied extensively in the literature. In all of these analyses, some of the standard assumptions used for circuit-switched networks are employed: The connection requests arrive at each node according to a Poisson process with rate $\gamma$, and the destination node for each connection request is uniformly distributed among all remaining nodes. The connection holding times are exponentially distributed with mean $1 / \mu$.

There are several factors that make the analysis of the blocking probability more difficult for WS networks compared to conventional circuit-switched networks. Some of these issues are summarized below.

- Link independence assumption: The standard assumption used in the analysis of circuit-switched networks is the link independence assumption, which states that the events on different links are independent and, hence, the blocking probability for a path can be expressed in product form [37], [56]. Although this assumption is shown to be accurate in various circuit-switched networks, the correlation between wavelength utilizations on adjacent links is strong in WS networks due to low traffic granularity. The blocking probability computed by employing the independence assumption is significantly overestimated, especially when the network topology is sparsely connected [37].

- Wavelength independence assumption: It is assumed that the individual wavelengths are utilized with a fixed probability independent of the utilizations of other wavelengths on the same link [37], [57]. Similar to the link independence assumption, the wavelength independence assumption leads to the overestimation of the blocking probability.

- Effect of wavelength selection algorithms: The distribution of the traffic on individual wavelengths depends on 
the wavelength selection algorithm. Most of the analyses in the literature assume identically distributed load on all wavelengths on the same link, which is true for a load-balancing wavelength-assignment algorithm such as the random wavelength selection [37], [49], [57], [56], [51]. However, as was pointed out earlier, wavelength packing type algorithms, such as the first-fit and mostused, achieve much smaller blocking probabilities. The analytical models for wavelength packing algorithms are more complicated and have large computational complexity [58], [59].

- Computational complexity: Due to the recursive nature of more complex algorithms, the penalty for including the dependence of link and wavelength loads in the model is additional computational complexity. For this reason, the computational results for more accurate models have been confined to small and symmetric networks. Since the link and wavelength independence assumptions and the wavelength selection algorithm affect the blocking probability substantially, simple analytical models have mostly been used for identifying the critical parameters in network topology design and wavelength/route assignment algorithms.

Kovacevic and Acampora [37] proposed one of the earlier analytical models to evaluate the benefits of wavelength converters. The model makes both the link and wavelength independence assumptions. It is also assumed that the random wavelength assignment is used in conjunction with the fixed shortest path routing. The path blocking probability in a network is computed using the reduced load approximation [38], [60], and Erlang fixed point equations are used to compute the link blocking probability in a recursive algorithm. Due to the independence assumptions the model is valid for only densely connected networks, and it significantly overestimates the blocking probability, especially for rings and sparsely connected meshes.

Barry and Humblet [57] refined the link independence assumption to incorporate the load dependence on successive links. A Markovian link correlation model is used: given the state of hop $i-1$, the load on hop $i$ is statistically independent of the loads on hops $1, \cdots, i-2$. The path blocking probability is computed assuming the random wavelength assignment algorithm. There are several findings that are revealed by this model. First, the wavelength conversion gain $G_{u}$ for a path increases with the number of hops $H$. For a fixed $H$, $G_{u}$ increases rapidly with the number of wavelengths $W$, and then levels off. More interestingly, the interference length parameter $L$, defined as the average number of hops shared by two intersecting paths, is identified as a critical parameter: the conversion gain $G_{u}$ decreases with $L$.

The interference length depends on the topology on the network as well as the routing algorithm. These dependencies are summarized below.

- For a unidirectional ring network with $N$ nodes, the interference length is large: $L=N / 2$.

- For a fully connected network with $N$ nodes and shortest path routing, the interference length is minimum: $L=1$.

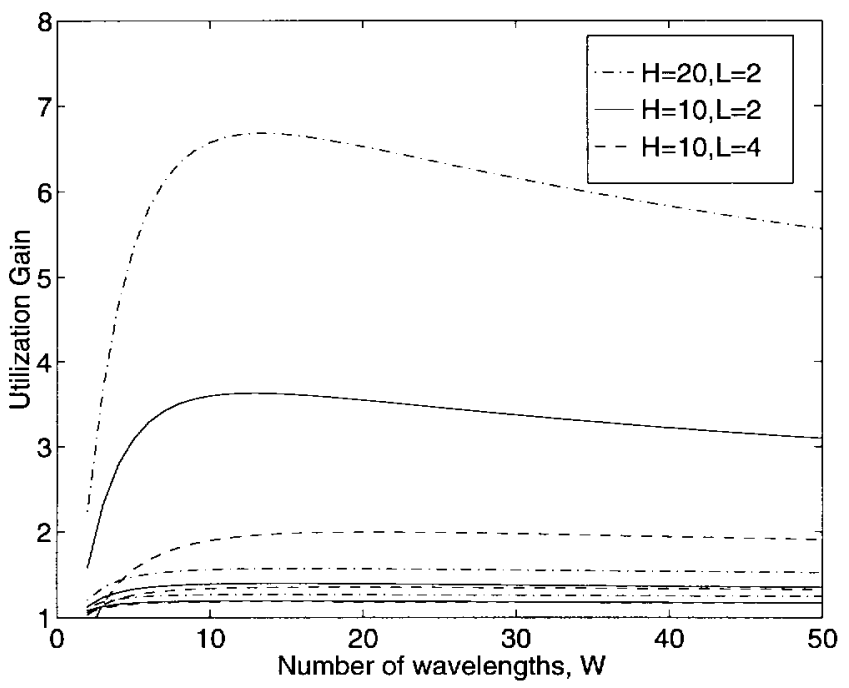

Fig. 6. $G_{u}$ versus the number of wavelengths for different values of $H$ and $L$ with $F=1,5,10$. The utilization gain decreases with increasing $F$.

- As shown in Fig. 8, the interference length for a fairly connected mesh network with alternate routing decreases as the path set gets larger [50].

This model is improved further to take links with multiple fibers into account [44], [49]. The predicted utilization gain for a path as $P_{B} \searrow 0$ is given by

$$
G_{u} \approx\left(\frac{H^{1-(1 / W)}}{L}\right)^{1 / F} \leq\left(\frac{H}{L}\right)^{1 / F}
$$

where $F$ is the number of fibers per link. It is observed from (1) that for large $W$, the wavelength conversion gain depends on $H / L$, called the effective path length by Barry and Humblet [57]. This explains the low $G_{u}$ values in the ring topology independent of the ring size for which $H / L \leq 2$, and the high values of conversion gain in the mesh-torus topology which has a large value of $H / L$ [37], [51]. The dependence of $G_{u}$ on $H, L, W$, and $F$ is demonstrated in Fig. 6 [44].

Strongest dependence of $G_{u}$ is on the number of fibers. The wavelength conversion gain drops exponentially with $1 / F$. Assuming that the number of wavelengths is limited by the physical constraints of the fiber and the other components in the transport network (such as optical amplifiers and optical filters), the number of fibers will increase as more and more traffic is carried over the transport network. Hence, the benefits of wavelength conversion will diminish with increasing traffic demand.

The dependence of $G_{u}$ on $F$ is shown in Fig. 7 obtained via simulations. Also plotted are the curves representing the exponential decrease with $F$, i.e., $G \sim(H / L)^{(1 / F)}$ [44], [49]. We observe that the exponential model overestimates the conversion gain and is fairly accurate at moderate values of $F$. Its accuracy diminishes as $F$ gets larger.

As was pointed out in Section II-B1), adaptive routing algorithms, such as LLR, increase the wavelength conversion gain. An intuitive reason for this is the increase in average path length. Furthermore, the interference length $L$ for a path decreases as the path set gets larger with an increasing number 


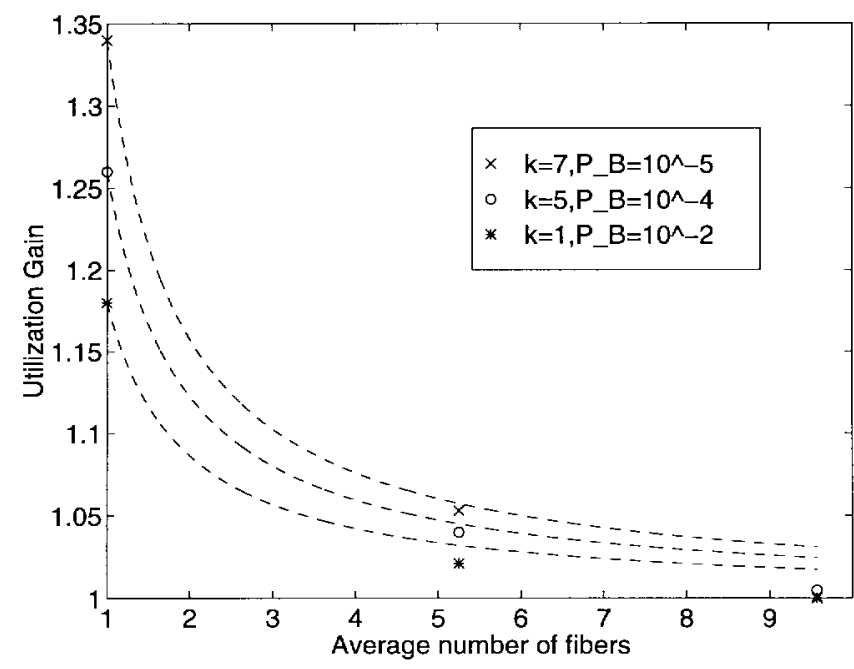

Fig. 7. $G_{u}$ versus the number of fibers for different values of $k$ and $P_{B}$.

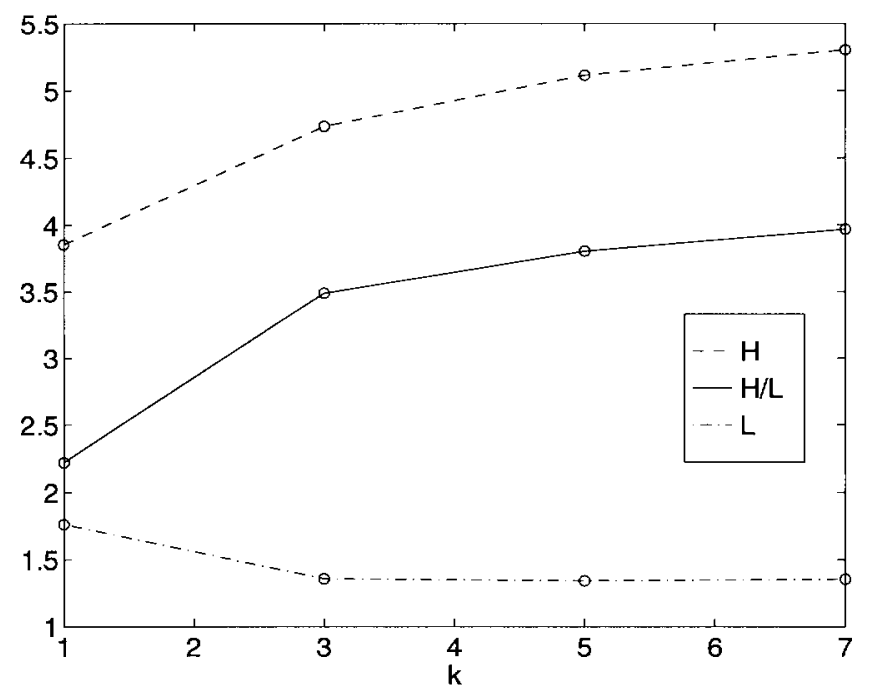

Fig. 8. $H, L$, and $H / L$ versus the number of alternate paths with LLR.

of alternate paths. These two trends result in an increase of the effective path length $H / L$ with $k$ as shown in Fig. 8.

A generalized reduced load approximation is used by Birman [56] to compute the blocking probability for the LLR algorithm with a random wavelength assignment algorithm in a fully connected network. The model uses the link independence assumption. The accuracy of the model is generally good, but it deteriorates at low utilizations. Furthermore, the large computational complexity of the generalized reducedload approximation considerably limits the size of the network to be analyzed (a study for a network of four nodes is given).

A computationally less intensive model for the fixed shortest path routing with the random wavelength assignment algorithm is presented by Subramaniam et al. [51]. This model does not make either link or wavelength independence assumptions, but instead uses the Markovian correlation model. The effect of blocking on the link arrival rates is not taken into account, i.e., the reduced load approximation is not used. The accuracy of the model is shown to be good with simulations for the ring and mesh-torus topologies.
There are recent analytical models for evaluating the blocking probability for WS networks with alternate routing and first-fit wavelength selection [53]. This model, however, does not consider the peakedness of the overflow traffic, and instead assumes that the overflow traffic is Poisson. Recently, analytical models for wavelength selection algorithms with lower blocking probabilities have been proposed [58], [59]. The overflow model is proposed by Karasan and Ayanoglu [58] to compute the blocking probability for the first-fit algorithm with fixed routing. The model uses the reduced load approximation [60] to compute link arrival rates more accurately. It is assumed that all the incoming Poisson traffic is offered to wavelength 1 . The traffic that cannot be carried over wavelength 1 (overflow traffic) is offered to wavelength 2 , and so on. Although the first offered traffic is Poisson, the overflow traffic is not Poisson, and the equivalent random method [38], [61] is used to compute the blocking probability by taking the peakedness of the non-Poisson traffic into account. Because of the iterative nature of the algorithm its computational complexity is high. The performance of the model is evaluated for a mesh-torus network with 16 nodes. The model is very accurate at medium and high loads, and it slightly overestimates the blocking probability at low loads. The model shows that, compared to the random assignment, the first-fit algorithm reduces the blocking probability.

3) Fairness and Admission Control: Another issue addressed in the literature is the dependence of the blocking probability on the path length in WS networks. Since wavelength conflicts are more probable for longer paths, the difference between the blocking probabilities with and without wavelength converters is largest for long paths. Meanwhile, wavelength interchanging networks admit more long paths which utilize more network resources. This results in higher blocking probability for short paths compared to those in WS networks [37], [50], [62].

There are two proposed algorithmic solutions to increase the fairness of the blocking probability as a function of the path length for WS networks. The first one is similar to the trunk reservation mechanism used for alternate routing in circuitswitched telephone networks [38], [60]. The trunk reservation protects the traffic on the direct link path from the overflow traffic from other direct link paths. This is accomplished by rejecting the overflow traffic if the utilization on the direct link path is above a threshold. In the protecting threshold technique proposed by Birman and Kershenbaum [62], the traffic on long paths in a WS network are protected from the traffic on onehop paths. The one-hop traffic is admitted only when the link utilization is below a threshold and, hence, the fairness of the blocking probability is increased.

Another algorithm to increase the fairness is presented by Harai et al. [63] which uses limited alternate paths such that longer paths have a larger number of alternates. By limiting the number of alternate paths for short connections, more connections requiring long paths can be accommodated. Both of these algorithms regulate the admission of connection requests by checking the network utilization and path length of the requested connection in order to improve the fairness of blocking for WS networks. 
While these two algorithms exercise the connection admission control to improve fairness, the overall blocking probability is increased. The general problem of admission control for WS networks in order to achieve low blocking probability while sustaining a high level of fairness has not yet been studied. The admission control problem is completely opposite for the wavelength-interchanging networks, where short paths have to be protected from long paths in order to reduce the average blocking probability. The blocking performances of WS and WI networks need to be compared when admission control algorithms that are separately optimized for each network are used.

\section{Transport Network Restoration}

One of the main functions of optical networks is to perform restoration after failures. Currently, different network restoration techniques have been implemented for different transport network technologies. In the SONET network, protection mechanisms based on self-healing rings and mesh restoration techniques using digital crossconnect systems (DCS's) are used against network failures. In the ATM network, virtual path (VP) crossconnects will be utilized for network restoration.

There are several problems with the multilayer restoration architectures. First, designing the interworking mechanisms between restoration mechanisms at different layers may be difficult. In particular, the problem of determining in which layer the restoration process starts, and when and to which layer the restoration activity is passed needs to be solved. Second, with the wide deployment of WDM systems in the transport network, the number of paths affected by a link failure is increased considerably with ATM networks since the VP's do not have any bandwidth hierarchy. Finally, and more importantly, when the optical transport network is used for restoration most of the restoration resources will be shared among different service layers, resulting in a more efficient utilization of restoration capacity.

The role of wavelength converters in optical transport network restoration has been studied extensively. These comparisons were made for centralized and preplanned restoration algorithms, and the number of wavelengths required for $100 \%$ protection against single-link failures with and without wavelength converters are computed [40], [41], [43], [47]. The heuristic WDM network design algorithms in these studies have been extended to design networks with excess resources for restoration. The restoration algorithm used in all these papers are end-to-end algorithms, i.e., all the paths affected by a link failure are rerouted between their source and destination nodes. The new path may share some or all of the links on the original path except the failed link. There are two variations in this algorithm for WS networks. The restoration path can either be assigned a new wavelength, i.e., wavelength tunable (WT), or it must use the same wavelength with the original path, i.e., wavelength nontunable (WNT).

The performances of these algorithms have been evaluated and compared with the restoration algorithms for the wavelength interchanging networks. The ratios of the number of wavelengths for a 15-node mesh network obtained by
Nagatsu et al. [40] are 15-20\% with WT networks and about $40 \%$ with WNT networks. Simulation studies for a polygrid network have shown that the ratio decreases with the network size, largely due to increased wavelength reusability in different parts of the network as the number of nodes increases [41]. Studies for other networks such as the European optical network with 19 nodes, ARPANET with 20 nodes, and NSFNet with 14 nodes produced similar results [43], [47].

The restoration problem for transport networks with a limited number of wavelengths is addressed by Nagatsu et al. [48]. The ratios of the number of optical crossconnect ports with and without wavelength conversion for a polygrid network are: 1) $30-40 \%$ with $W=8$; 2) $50-60 \%$ with $W=16$; and 3) $70-80 \%$ with $W=32$. The ratio increases with $W$, and decreases with the traffic volume. Those ratios considering restoration are larger than the case with unlimited number of wavelengths and larger than the ratios considering only service paths. However, there are two important points. First, these numbers are calculated using heuristics since the optimum solution is difficult to obtain. Due to the joint assignment of routes and wavelengths, the design problem for WS networks is much more difficult, and the solutions given by the heuristic algorithm are further away from the optimum solution. Second, the service and restoration path problems are separately solved in [48]. Better solutions will be obtained if these two problems are combined and jointly solved which will result in more resource sharing between service and restoration layers. This technique is likely to improve the results more for the wavelength-selective case since the constraints put by the independently designed service layer create many wavelength conflicts.

Designing new heuristic WDM network-design algorithms for jointly solving the service and restoration layer problems is very important for the successful deployment of WDM restoration techniques in transport networks.

\section{Sparse Wavelength Conversion}

So far our discussion on the performance of optical networks has been confined to networks that have wavelength conversion at all ports in all optical crossconnects. On the other hand, there may be technological and economic considerations for using wavelength conversion at a subset of nodes (sparse conversion) or using wavelength converters that can convert a wavelength into a subset of wavelengths (limited conversion).

Sparse conversion has the economic advantage of reducing the high cost of wavelength converters in the network. The blocking performance of wavelength converters has been studied for mesh and ring networks by Subramaniam et al. [51]. It is assumed that a node is capable of wavelength conversion with probability $q$ independent of other nodes. It is shown for a mesh-torus network that the blocking probability reduces rapidly with the conversion density $q$ and then levels off.

The model introduced by Subramaniam points to the usefulness of wavelength conversion at a small number of nodes for some topologies. The selection of the nodes where converters are placed, however, is not addressed. The converter placement problem, as well as developing good routing and wavelength selection algorithms for these hybrid optical networks, are cur- 
rently open problems. One heuristic for converter placement is to select the nodes which carry a large number of long paths since longer paths are more susceptible to wavelength conflicts.

One other factor in selecting the converter locations is to simplify the network management. Wauters et al. [64] considered optoelectronic converters installed at the boundaries between subnetworks that constitute a national-scale transport network. Connections that pass through multiple subnetworks can be regenerated at the boundaries, and the wavelength selection problem is solved only within a subnetwork. This technique reduces the complexity of the wavelength routing and assignment algorithm and network management is simplified. Furthermore, the blocking probability is reduced: by placing wavelength converters at 6 out of 19 nodes in the European optical network, the blocking performance is almost identical to the wavelength interchanging network [64].

Nagatsu et al. [65] exercise the optoelectronic wavelength conversion to reduce the number of ports in the optical transport network design problem. An algorithm is employed to reassign the wavelengths in such a way that the number of interoffice ports can be reduced by dropping the connection to an electronics based crossconnect at an intermediate node. The connection is regenerated at a different wavelength and is added back to the optical transport network. The algorithm allows, at most, one such conversion for each connection. Since the conversion can be performed electronically at any node, there is no converter placement problem. The ratio of ports (WS versus WI) in a $5 \times 10$ polygrid network is reduced from $10-15 \%$ to $5-7 \%$ at $W=8$. The economical implications of this technique, however, have to be studied further since the cost of the electrical portion of the transport network increases due to an increased number of electrical crossconnect ports.

Bala et al. [23] proposed sparse wavelength conversion for interconnected ring networks to improve the fairness of the blocking probability as a function of the path length.

\section{E. Limited Wavelength Conversion}

Limited wavelength conversion is motivated by technological considerations. Experimental results show that the conversion bandwidth of four-wave mixing wavelength converters is limited to $1 \mathrm{THz}$ on either side of the input wavelength [28], [29]. Assuming a channel spacing of 100 $\mathrm{GHz}$, the four-wave mixing wavelength converter is able to convert to about 20 different wavelengths. The conversion bandwidths of crossphase and crossgain wavelength converters are 2 and $3 \mathrm{THz}$, respectively [28], [29]. Considering that point-to-point WDM transmission systems with 32 and more wavelengths will be available in the near future [1], [66] and systems with more wavelengths will be achievable in the next five years, the assumption that any wavelength can be converted to any other wavelength does not hold unless optoelectronic conversion is used.

The blocking probability for an optical network with limited range wavelength converters has been studied by Yates et al. [67] for ring and mesh-torus topologies with fixed shortest path routing. Harai et al. [59] used a hypercube network to study limited conversion with fixed shortest path routing and a first-fit wavelength selection algorithm. An algorithm is used in [67] to minimize the number of wavelength conversions and to pack wavelengths. It is shown that with a conversion bandwidth that covers only $25 \%$ of the whole transmission bandwidth, the blocking probability is almost identical to the one with full-range conversion.

Gerstel et al. [68] computed the maximum number of carried connections on a ring network as a function of the conversion degree $d$. The conversion degree is the number of output wavelengths that an input wavelength can be converted. In the static traffic case, the limited wavelength conversion with $d=2$ has a throughput equal to the one with full conversion. Currently, however, with dynamic traffic demand, there is no available algorithm that achieves a guaranteed high throughput with limited conversion.

Another study on limited wavelength conversion has a wavelength interchanging crossconnect architecture where converters are not dedicated to individual channels [22]. A smaller number of converters are shared among different channels in order to lower the switch cost. The performance of this crossconnect is evaluated using the ARPA network with 21 nodes. The blocking probability with a crossconnect which has converters at only $25 \%$ of the total number of channels is almost equal to the one with full conversion.

\section{F. Multilayer Crossconnect Advantages}

Significant cost reductions can be achieved by dividing the functionality of a wavelength crossconnect between one that crossconnects fibers (FXC) and one that crossconnects wavelengths (WSXC or WIXC). As the traffic increases, more and more fibers will go through the network nodes in an express mode, without adding or dropping any wavelengths and with all the wavelengths on an incoming fiber going on to the same fiber. When this is the case, passing all such wavelengths through wavelength demultiplexing and multiplexing is not necessary. Thus, the number of wavelengths going through WSXC's or WIXC's can be significantly reduced. As the network traffic increases, this two-layer technology will become more attractive. In addition, it is possible to implement network restoration algorithms that have a combination of FXC's and WSXC's for provisioning purposes but employ only FXC's for restoration against failures. These networks combine a reduction in the cost of crossconnects with fast network restoration.

Generalizing this idea, from a cost minimization viewpoint, the best crossconnect architecture can be obtained by combining multiple layers of crossconnects, each operating at a different granularity, thus achieving the best packing of wavelengths onto fibers and the best packing of electrical tributaries onto wavelengths. This architecture is shown in Fig. 9. Thus, such a crossconnect has an FXC at its lowest layer which crossconnects express fibers directly. Eventually, as the traffic increases, this layer may terminate (or initiate) fibers locally. The middle layer consists of a wavelength crossconnect (WSXC or WIXC) which demultiplexes fibers into wavelengths and routes wavelengths from a fiber separately 


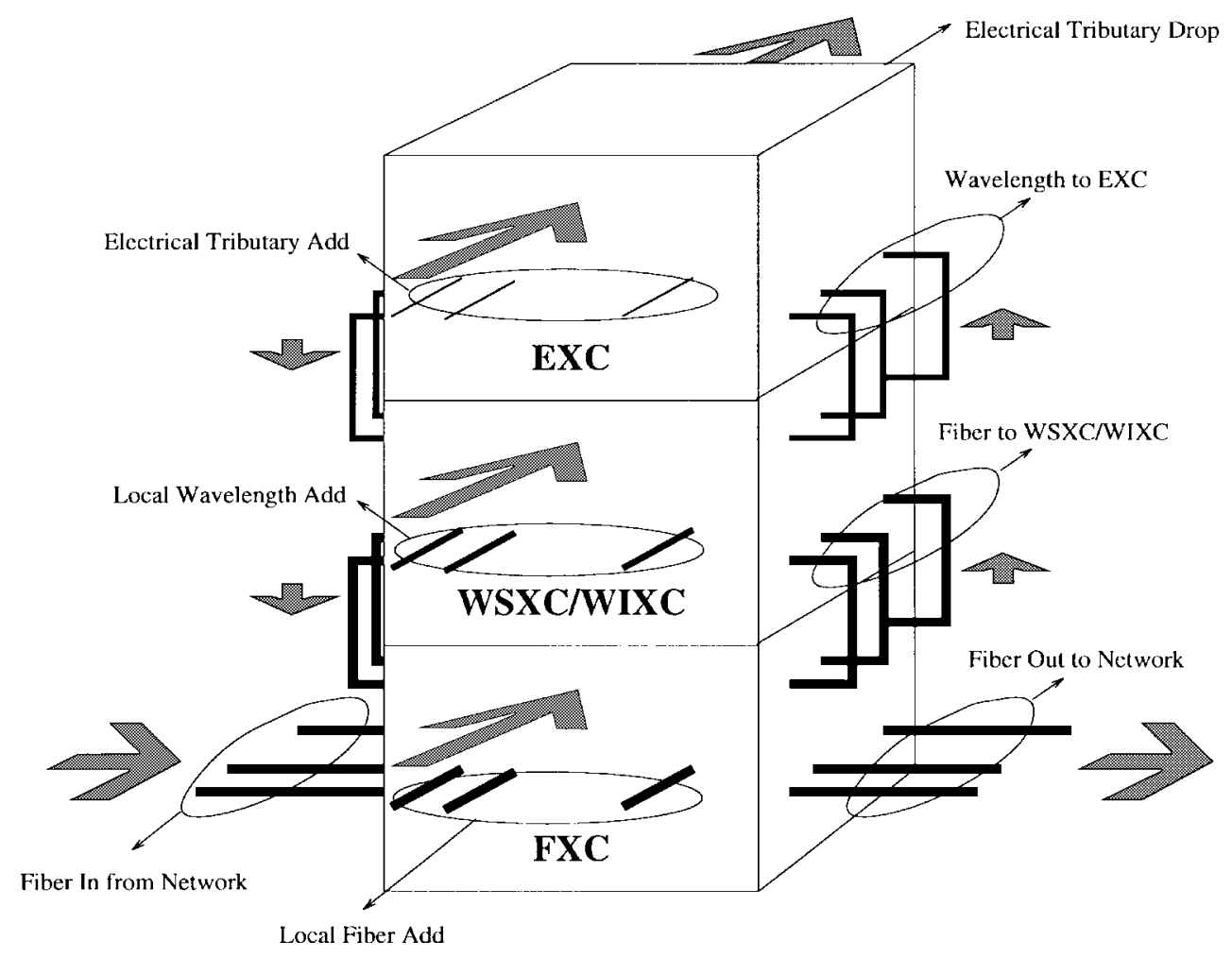

Fig. 9. Multilayer crossconnect architecture.

to their destinations. Different wavelengths destined for the same node are then multiplexed onto fibers. This layer includes a wavelength add/drop multiplexer for local traffic when the local traffic reaches wavelength granularity. The topmost layer is an electrical crossconnect. This layer is used to pack each wavelength as tightly as possible with individual electrical tributaries. Again, this layer has add/drop connections for the local traffic.

\section{SUMmARY AND CONCLUSIONS}

Although the WDM technology has been available commercially for only a few years, its deployment has taken place at a surprisingly high speed. Already, all major long-distance network providers in the United States employ WDM in their backbone networks. There is no doubt that WDM as a pointto-point transmission technology will continue to be deployed in the WAN backbone at increasing speeds. It is generally expected that WDM networking will be next in the deployment of WDM. Thus, the design and analysis of WDM networks (which require methods different and more sophisticated than those used in conventional circuit-switched networks), has become very significant. In this paper we have outlined WDM network design and performance analysis results from the literature.

There has been a significant debate on the utility of wavelength conversion in WDM networks. Wavelength conversion reduces the probability of blocking and thus increases utilization. Quantifying the wavelength conversion gain has been attempted by many authors and various conclusions have been drawn, sometimes with conflicting results. A summary of results from the literature is provided in Tables II and III for the static and the dynamic problems, respectively. We finalize this paper with a list of open problems.

1) Most of the existing routing, wavelength selection, and other OAM algorithms in the literature are centralized. For simplicity and scalability purposes, distributed algorithms are desirable. This problem has not been attacked so far in the open literature.

2) Most of the design, analysis, and simulation work in the literature is for mesh or ring architectures. Networks with layered topologies, such as rings of rings and mesh of rings, are being deployed. Many simple network problems become difficult in these new topologies. New results are needed from a WDM network design and analysis perspective in these layered topologies.

3) WDM networks with the layered node architecture as discussed in Section II-F are very attractive since they enable a high degree of grooming and, thus, packing of each wavelength and each fiber as efficiently as possible. Again, however, much work is needed to understand how to design, separate various tasks (e.g., restoration) among various layers, and operate these networks.

4) Many vendors are beginning to introduce WDM systems with a large number of wavelengths, such as 40 or 80 . Theory indicates [e.g., see (1)] that the gain will slightly increase but quickly saturate by increasing the number of wavelengths. It can be expected that simulations presented in this paper show a slight gain toward wavelength conversion when repeated with a large number of wavelengths. In that case, however, the more important issue is the difficulty of scaling all-optical networking technologies to a large number of wavelengths. 
TABLE II

Wavelength Conversion Gains for Network Design Algorithms with Static Demand. All Studies Were Done for the Mesh Topology. WT: Wavelength Tunable, WNT: WaVelength Nontunable

\begin{tabular}{|c|c|c|c|c|c|c|}
\hline Algorithm & Reference & \# Nodes & Objective & $\# \lambda$ & Gain & $\begin{array}{l}\text { Gain with } \\
\text { Restoration }\end{array}$ \\
\hline Longer Paths First & [39] & 10 & Minimize $\# \lambda$ & unbounded & $2 \%(\# \lambda)$ & NA \\
\hline Longer Paths First & {$[40,41,42]$} & $15-50$ & Minimize $\# \lambda$ & unbounded & $<4 \%(\# \lambda)$ & $\begin{array}{l}15-20 \% \text { (\# } \lambda), \text { WT } \\
40 \%(\# \lambda), \mathrm{WNT}\end{array}$ \\
\hline Minimum Cutset & [43] & $\begin{array}{c}14-20 \\
21\end{array}$ & Minimize $\# \lambda$ & unbounded & $\begin{array}{c}0 \%(\# \lambda) \\
<16 \%(\# \lambda)\end{array}$ & $\begin{array}{l}<18 \%(\# \lambda) \\
<37 \%(\# \lambda)\end{array}$ \\
\hline Heuristic & [45] & 20 & $\begin{array}{c}\text { Maximize } \\
\text { carried traffic }\end{array}$ & 100 & $\begin{array}{l}100-200 \% \text { (\# blocked } \\
\text { connections) }\end{array}$ & NA \\
\hline $\begin{array}{c}\text { Multicommodity Flow } \\
\text { Flow Formulation }\end{array}$ & {$[47]$} & 19 & $\begin{array}{l}\text { Maximize carried } \\
\text { traffic (all paths) }\end{array}$ & $9-20$ & $\begin{array}{c}0 \% \text { (\# blocked } \\
\text { connections) }\end{array}$ & $\begin{array}{c}5 \% \text { (\# blocked } \\
\text { connections) }\end{array}$ \\
\hline $\begin{array}{c}\text { Multicommodity Flow } \\
\text { Path Formulation }\end{array}$ & [47] & 19 & $\begin{array}{l}\text { Maximize carried } \\
\text { traffic ( } k \text { paths) }\end{array}$ & 9-20 & $\begin{array}{c}0 \% \text { (\# blocked } \\
\text { connections) }\end{array}$ & $\begin{array}{c}5 \% \text { (\# blocked } \\
\text { connections) }\end{array}$ \\
\hline Heuristic & {$[48]$} & 15 & Minimize \# ports & $\begin{array}{c}8 \\
32\end{array}$ & $\begin{array}{l}15 \% \text { (\# ports) } \\
35 \% \text { (\# ports) }\end{array}$ & $\begin{array}{l}30-40 \% \text { (\# ports) } \\
70-80 \% \text { (\# ports) }\end{array}$ \\
\hline
\end{tabular}

TABLE III

Wavelength Conversion Gains with Routing and Wavelength Assignment Algorithms for Dynamic Demand. The Objective Is to Minimize $P_{B}$. Uniform Traffic, Poisson Arrivals, and Exponentially Distributed Holding Times Were Assumed in All Studies. RA: Routing Algorithm, WSA: Wavelength Selection Algorithm, SP: Shortest Path, FF: First-Fit, $M \Sigma$ : MaX-Sum, ALT: Alternate Routing, LLR: Least Loaded Routing, IP: Integer Programming, $P_{B}=$ Blocking Probability, $\rho=$ Average Utilization Per Link Per Wavelength

\begin{tabular}{|c|c|c|c|c|c|c|c|c|}
\hline $\mathrm{RA}$ & WSA & Reference & Arrivals & Topology & \# Nodes & \# Fibers & $\# \lambda$ & Gain \\
\hline SP & FF & [37] & Single & $\begin{array}{c}\text { Ring } \\
\text { Mesh-Torus }\end{array}$ & $\begin{array}{c}100 \\
101 \times 101\end{array}$ & 1 & $\begin{array}{c}5 \\
100 \\
5\end{array}$ & $\begin{array}{c}G_{u}<1.08\left(P_{B}=10^{-4}\right) \\
G_{u}<1.25\left(P_{B}=10^{-4}\right) \\
G_{p}=10^{4}(\rho=0.12) \\
G_{u}=5\left(P_{B}=10^{-3}\right)\end{array}$ \\
\hline $\mathrm{SP}$ & FF & [51] & Single & $\begin{array}{c}\text { Ring } \\
\text { Mesh-Torus }\end{array}$ & $\begin{array}{c}100 \\
101 \times 101\end{array}$ & 1 & $\begin{array}{c}5 \\
10 \\
8\end{array}$ & $\begin{array}{c}G_{p}<1.25(\rho=0.2) \\
G_{p}<10(\rho=0.1) \\
G_{p}=10^{8}(\rho=0.03)\end{array}$ \\
\hline SP & $\mathrm{M} \Sigma$ & [53] & Single & $\begin{array}{c}\text { Ring } \\
\text { Mesh-Torus }\end{array}$ & $5 \times 5$ & $\begin{array}{c}1 \\
10 \\
1 \\
3\end{array}$ & $\begin{array}{l}50 \\
50 \\
50 \\
50\end{array}$ & $\begin{aligned} G_{p} & <15 \\
G_{p} & <7 \\
G_{p} & <10 \\
G_{p} & <2.5\end{aligned}$ \\
\hline ALT & FF & [45] & Single & Mesh & $\begin{array}{c}16 \\
1000 \\
\end{array}$ & $\begin{array}{l}1 \\
1 \\
\end{array}$ & $\begin{array}{r}10-32 \\
10-32 \\
\end{array}$ & $\begin{array}{l}G_{u} \approx 1.0\left(P_{B}=10^{-2}\right) \\
G_{u}=1.4\left(P_{B}=10^{-2}\right)\end{array}$ \\
\hline \multicolumn{2}{|c|}{ LLR } & [50] & Single & Mesh & 30 & $\begin{array}{c}1 \\
5 \\
10\end{array}$ & $\begin{array}{l}8 \\
8 \\
8\end{array}$ & $\begin{array}{c}G_{u}=1.3\left(P_{B}=10^{-4}, k=7\right) \\
G_{u}=1.07\left(P_{B}=10^{-4}, k=7\right) \\
G_{u}=1.005\left(P_{B}=10^{-4}, k=7\right)\end{array}$ \\
\hline & $P$ & [55] & Batch & Mesh & 30 & 5 & 8 & $\begin{array}{c}G_{p}=2.0 \text { (limited reconfig.) } \\
G_{p}=1.0 \text { (full reconfig.) }\end{array}$ \\
\hline
\end{tabular}




\section{REFERENCES}

[1] A. H. Gnauck, A. R. Chraplyvy, and R. W. Tkach, "One terabit/s transmission experiment," in Proc. IEEE OFC'96, 1996, vol. 2, pp. 407-410.

[2] G. Kotelly, "Worldwide fiber-optic markets to expand unabated," $J$. Lightwave Technol., vol. 13, pp. 6-8, Dec. 1996.

[3] S. B. Alexander et al., "A precompetitive consortium on wide-band alloptical networks," J. Lightwave Technol., vol. 11, pp. 714-735, May 1993.

[4] I. P. Kaminow et al., "A wide-band all-optical WDM network," IEEE J. Select. Areas Commun., vol. 14, pp. 780-799, June 1996.

[5] C. Brackett et al., "A scalable multiwavelength multihop optical network: A proposal for research on all-optical networks," J. Lightwave Technol., vol. 11, pp. 736-753, May 1993.

[6] G.-K. Chang et al., "Multiwavelength reconfigurable WDM/ATM/SONET network testbed," J. Lightwave Technol., vol. 14, pp. 1320-1340, June 1996.

[7] R. E. Wagner et al., "MONET: Multiwavelength optical networking," J. Lightwave Technol., vol. 14, pp. 1349-1355, June 1996.

[8] Available: http://www-phys.llnl.gov/H\_Div/photonics/NTONC

[9] G. R. Hill et al., "A transport network layer based on optical network elements," J. Lightwave Technol., vol. 11, pp. 667-679, May 1993.

[10] G.-D. Khoe et al., "Coherent multicarrier technology for implementation in the customer access," J. Lightwave Technol., vol. 11, pp. 695-713, May 1993.

[11] P. J. Smith et al., "Evolution scenarios for optical telecommunication networks using multiwavelength transmission," Proc. IEEE, vol. 81, pp. 850-857, Nov. 1993.

[12] P. Chidgey, "Multi-wavelength transport networks," IEEE Commun. Mag., vol. 32, pp. 28-35, Dec. 1994.

[13] M. J. O'Mahony et al., "The design of a European optical network," $J$. Lightwave Technol., vol. 13, pp. 817-828, 1995.

[14] S. Johansson, "Transport network involving a reconfigurable WDM network layer-A European demonstration," J. Lightwave Technol., vol. 14, 1996

[15] Available: http://www.tel.fer.hr/josip/marie/COST239.htm

[16] D. W. Smith and G. R. Hill, "Optical processing in future coherent networks," in Proc. IEEE GLOBECOM 1987, Tokyo, Japan, pp. $678-683$.

[17] B. Strebel and G. Heydt, "Multi-channel optical carrier frequency technique," in Proc. IEEE GLOBECOM 1987, Tokyo, Japan, pp. 684-688.

[18] S. Johansson, M. Lindblom, P. Granestrand, B. Lagerström, and L. Thylén, "Optical cross-connect system in broadband networks: System concept and demonstrators description," J. Lightwave Technol., vol. 11, pp. 688-694, May 1993

[19] K.-I. Sato, S. Okamoto, and H. Hadama, "Optical path layer technologies to enhance B-ISDN performance," in Proc. IEEE ICC '93, May 1993 pp. $1300-1307$.

[20] P. Dumortier, F. Masetti, and M. Sotom, "Guidelines for scalable optical telecommunication networks," in Proc. IEEE GLOBECOM '95, Nov. 1995, pp. 1012-1017.

[21] K.-I. Sato, "Photonic transport network OAM technologies," IEEE Commun. Mag., vol. 34, pp. 86-94, Dec. 1996.

[22] K.-C. Lee and V. O. K. Li, "A wavelength-convertible optical network," J. Lightwave Technol., pp. 962-970, May 1993.

[23] K. Bala, E. Boulliet, and G. Ellinas, "Benefits of 'minimal' wavelength interchange in WDM rings," in Tech. Dig. IEEE OFC, Dallas, TX, Feb. 1997, pp. 120-121.

[24] H. Inose, "Blocking probability and junktor capacity of a three stage time-division switching network," J. Inst. Electr. Commun. Eng. Jpn., vol. 44, pp. 934-941, June 1961.

[25] _ , An Introduction to Digital Integrated Communications Systems. Tokyo, Japan: Univ. of Tokyo Press, 1979.

[26] R. J. Chaupis and A. E. Joel, Jr., Electronics, Computers, and Telephone Switching, vol. 2. Amsterdam, The Netherlands: Elsevier, 1990.

[27] C. Dragone, C. A. Edwards, and R. C. Kistler, "Integrated optics $N \times N$ multiplexer on silicon," IEEE Photon. Technol. Lett., vol. 3, pp. 896-899, Oct. 1991.

[28] S. J. B. Yoo, "Wavelength conversion technologies for WDM network applications," J. Lightwave Technol., vol. 14, pp. 955-966, June 1996.

[29] S. J. B. Yoo, C. Caneau, A. Rajhel, J. Ringo, R. Bhat, M. A. Koza, M. Amersfoort, J. Baran, K. Bala, N. Antoniades, and G. Ellinas, "MONET Performance Milestone Rep.," Mar. 1997.

[30] J. Karuso, "IBM lights dark fiber," Communications Week, p. 1, Oct. 1996.

[31] F. J. Janniello, R. Ramaswami, and D. G. Steinberg, "A prototype circuit-switched multi-wavelength optical metropolitan-area network," J. Lightwave Technol., vol. 11, pp. 777-782, May 1993.
[32] W. C. Marra and J. Schesser, "Africa ONE: The africa optical network," IEEE Commun. Mag., vol. 34, pp. 50-57, Feb. 1996.

[33] J. Rendleman, "Sprint's cross-country link completed," Communications Week, p. 21, July 3, 1995

[34] E. Schroeder, "MCI completes new SONET rings," PC Week, Apr. 22, 1996.

[35] G. Kotelly, "AT\&T charts network growth over installed fiber plant," Lightwave, June 1997

[36] J. A. Bannister, L. Fratta, and M. Gerla, "Topological design of the wavelength division optical network," in Proc. IEEE INFOCOM '90, San Francisco, CA, June 1990, pp. 1005-1013.

[37] M. Kovacevic and A. Acampora, "Benefits of wavelength translation in all-optical clear-channel networks," IEEE J. Select. Areas Commun./J. Lightwave Technol., vol. 14, no. 5, pp. 868-880, 1996.

[38] A. Girard, Routing and Dimensioning in Circuit-Switched Networks. Reading, MA: Addison-Wesley, 1990

[39] I. Chlamtac, A. Ganz, and G. Karmi, "Lightpath communications: An approach to high bandwidth optical WAN's," IEEE Trans. Commun., vol. 40, pp. 1171-1182, July 1992.

[40] N. Nagatsu, Y. Hamazumi, and K. Sato, "Optical path accommodation designs applicable to large scale networks," IEICE Trans. Commun., vol. E78-B, no. 4, pp. 597-607, 1995.

[41] Y. Hamazumi, N. Nagatsu, S. Okamoto, and K. Sato, "Number of wavelengths required for constructing optical path network considering restoration,” Elec. Commun. Jpn., Pt. 1, vol. 78, no. 7, pp. 30-41, 1995.

[42] N. Nagatsu, Y. Hamazumi, and K. Sato, "Number of wavelength required for constructing large-scale optical path networks," Elec. Commun. Jpn., Pt. 1, vol. 78, no. 9, pp. 1-11, 1995.

[43] S. Baroni and P. Bayvel, "Wavelength requirements in arbitrarily connected wavelength-routed optical networks," J. Lightwave Technol., vol. 15, no. 2, pp. 242-251, Feb. 1997.

[44] G. Jeong and E. Ayanoglu, "Comparison of wavelength-interchanging and wavelength-selective cross-connects in multiwavelength all-optical networks," in Proc. IEEE INFOCOM, San Francisco, CA, Mar. 1996, pp. $156-163$.

[45] R. Ramaswami and K. N. Sivarajan, "Routing and wavelength assignment in all-optical networks," IEEE/ACM Trans. Networking, vol. 3, no. 5, pp. 489-500, 1995.

[46] R. K. Ahuja, T. L. Magnanti, and J. B. Orlin, Network Flows. Englewood Cliffs, NJ: Prentice-Hall, 1993.

[47] N. Wauters and P. Demeester, "Design of the optical path layer in multiwavelength cross-connected networks," IEEE J. Select. Areas Commun./J. Lightwave Technol., vol. 14, no. 5, pp. 881-892, 1996.

[48] N. Nagatsu, S. Okamoto, and K. Sato, "Optical path cross-connect system scale evaluation using path accommodation design for restricted wavelength multiplexing," IEEE J. Select. Areas Commun./J. Lightwave Technol., vol. 14, no. 5, pp. 893-902, 1996.

[49] R. Barry and D. Marquis, "An improved model of blocking probability in all-optical networks," in IEEE LEOS Summer Topicals Meeting, Keystone, CO, 1995, pp. 43-44.

[50] E. Karasan and E. Ayanoglu, "Effects of wavelength routing and selection algorithms on wavelength conversion gain in WDM optical networks," in Proc. IEEE GLOBECOM, London, U.K., Nov. 1996, pp. 299-305.

[51] S. Subramaniam, M. Azizoğlu, and A.K. Somani, "All-optical networks with sparse wavelength conversion," IEEE/ACM Trans. Networking, vol. 4 , pp. $544-557,1996$.

[52] A. Mokhtar and M. Azizoğlu, "Adaptive wavelength routing in alloptical networks," IEEE/ACM Trans. Networking, vol. 6, pp. 197-206, Apr. 1998.

[53] S. Subramaniam and R. Barry, "Wavelength assignment in fixed routing WDM networks," in Proc. IEEE ICC, Montreal, Canada, Nov. 1997, pp. $406-410$

[54] G. R. Ash, "Dynamic network evolution with examples from AT\&T' evolving network," IEEE Commun. Mag., vol. 33, pp. 26-39, July 1995.

[55] E. Karasan and E. Ayanoglu, "Performance comparison of reconfigurable wavelength selective and wavelength interchanging crossconnects in WDM transport networks," in Proc. 3rd IEEE/COMSOC Workshop on WDM Network Management and Control, Montreal, Canada, Nov. 1997.

[56] A. Birman, "Computing approximate blocking probabilities for a class of all-optical networks," IEEE J. Select. Areas Commun./J. Lightwave Technol., vol. 14, no. 5, pp. 852-857, 1996.

[57] R. A. Barry and P. A. Humblet, "Models of blocking probability in alloptical networks with and without wavelength changers," IEEE J. Select Areas Commun./J. Lightwave Technol., vol. 14, no. 5, pp. 858-867, 1996.

[58] E. Karasan and E. Ayanoglu, "Effects of wavelength routing and selection algorithms on wavelength conversion gain in WDM optical 
networks," IEEE/ACM Trans. Networking, vol. 6, pp. 186-196, Apr. 1998.

[59] H. Harai, M. Murata, and H. Miyahara, "Performance of all-optical networks with limited range wavelength conversion," in Proc. IEEE ICC, Montreal, Canada, 1997, pp. 416-421.

[60] K. W. Ross, Multiservice Loss Models for Broadband Telecommunication Networks. Springer-Verlag, 1995.

[61] J. Y. Hui, Switching and Traffic Theory for Integrated Broadband Networks. Norwell, MA: Kluwer, 1990.

[62] A. Birman and A. Kershenbaum, "Routing and wavelength assignment methods in single-hop all-optical networks with blocking," in Proc. IEEE INFOCOM, Boston, MA, Apr. 1995, pp. 431-438.

[63] H. Harai, M. Murata, and H. Miyahara, "Performance of alternate routing methods in all-optical switching networks," in Proc. IEEE INFOCOM, Kobe, Japan, Apr. 1997, pp. 516-524.

[64] N. Wauters, W. Van Parys, B. Van Caenegem, and P. Demeester, "Reduction of wavelength blocking through partitioning with wavelength converters," in Tech. Dig. IEEE OFC, Dallas, TX, Feb. 1997, pp. $122-123$.

[65] N. Nagatsu, S. Okamoto, and K. Sato, "Large scale photonic transport network design based on optical paths," in Proc. IEEE GLOBECOM, London, U.K., Nov. 1996, pp. 321-327.

[66] T. Dagres and J. P. Ryan, "The order of magnitude," Telecom Magazine, Apr. 1997.

[67] J. Yates, J. Lacey, D. Everitt, and M. Summerfield, "Limited-range wavelength translation in all-optical networks," in Proc. IEEE INFOCOM, San Francisco, CA, Mar. 1996, pp. 954-961.

[68] O. Gerstel, R. Ramaswami, and G. Sasaki, "Benefits of limited wavelength conversion in WDM ring networks," in Tech. Dig. IEEE OFC, Dallas, TX, Feb. 1997, pp. 119-120.
Ezhan Karasan (M'95), for photograph and biography, see this issue, p. 1079.

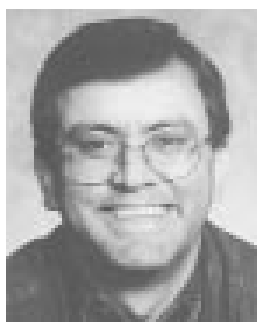

Ender Ayanoglu (S'82-M'85-SM'90-F'98) received the B.S. degree from Middle East Technical University, Ankara, Turkey, in 1980, and the M.S and $\mathrm{Ph} . \mathrm{D}$. degrees in electrical engineering from Stanford University, Stanford, CA, in 1982 and 1986, respectively.

Since 1986, he has been with Bell Labs. During 1990-1991 and 1992, he taught at Bilkent University, on leave from Bell Labs. During 1995-1997, he was the Long Distance Architecture Task Leader of the Multiwavelength Optical Networking (MONET) project. His current research interests are in multiwavelength optical networking, broadband integrated communications, wireless ATM, and general source and channel coding applications in communication networks.

Dr. Ayanoglu is the Vice Chair of the IEEE Communication Society Communication Theory Technical Committee and an Editor of the IEEE TRANSACTIONS ON COMMUNICATIONS for Communication Theory and Coding Applications. He received the IEEE Communications Society Stephen O. Rice Prize Paper Award in 1995 and the IEEE Communications Society Best Tutorial Paper Award in 1997. 